\title{
Avaliação de tecnologias de refúgio no cultivo de milho transgênico
}

\author{
Paolo Orlando Zancanaro(1), Elizandra Döring Buchweitz ${ }^{(1)}$, Arlindo Leal Boiça Junior ${ }^{(1)}$ e José Roberto Moro(1) \\ (1)Universidade Estadual Paulista, Faculdade de Ciências Agrárias e Veterinárias, Via de Acesso Prof. Paulo Donato Castellane, s/no, \\ CEP14884-900 Jaboticabal, SP. E-mail: paolozancanaro@gmail.com, elizandrabuchweitz@yahoo.com.br, aboicajr@fcav.unesp.br, \\ jrmoro@fcav.unesp.br
}

\begin{abstract}
Resumo - O objetivo deste trabalho foi comparar o uso de refúgio tradicional em áreas separadas com a tecnologia alternativa de refúgio pela mistura de sementes não transgênicas no saco ("refuge in the bag" - RIB) em diferentes proporções. Foram utilizados os híbridos comerciais transgênicos AG 7000YG e DKB 390YG com refúgios plantados com as respectivas cultivares convencionais. Avaliaram-se sete tratamentos: RIB com quatro proporções de mistura de sementes não transgênicas $(2,5,5,0,7,5$ e 10\%) no saco; refúgio tradicional, com $10 \%$ da área plantada exclusivamente com cultivar não transgênica; e área totalmente cultivada com plantas transgênicas ou totalmente com plantas não transgênicas. O híbrido DKB 390YG foi o mais produtivo. Para este híbrido, não foram observadas diferenças de produtividade entre os tratamentos, com exceção do controle inteiramente convencional, que produziu menos. Com o híbrido AG 7000YG, a tecnologia RIB com proporções de 5,0 e 7,5\% de sementes não transgênicas apresentou as maiores produtividades, significativamente superiores às do refúgio tradicional, que não diferiu do controle convencional. Nos tratamentos RIB, as diferenças nas intensidades de dano por Spodoptera frugiperda não se refletiram em diferenças na produtividade. O refúgio no saco é alternativa viável para substituir o método de refúgio utilizado atualmente.
\end{abstract}

Termos para indexação: Spodoptera frugiperda, Zea mays, mistura de sementes, refúgio no saco, resistência de insetos, "yieldgard".

\section{Evaluation of refuge technologies in transgenic maize crop}

\begin{abstract}
The objective of this work was to compare the traditional refuges in separated areas with the alternative technology of refuge provided by the mixture of conventional seeds in the bag (refuge in the bag, RIB) with different proportions. The commercial transgenic hybrids AG 7000YG and DKB 390YG were used with the refuges sown with their respective conventional cultivars. Seven treatments were evaluated: RIB with four mixing rates of nontransgenic seeds $(2.5,5.0,7.5$, and $10 \%)$; traditional refuge, with $10 \%$ of the area sown exclusively with nontransgenic cultivar; and entirely cultivated areas with transgenic or nontransgenic plants. The DKB 390YG hybrid was the most productive. For this hybrid, there were no yield differences between treatments, except for the control (entirely conventional), which had lower yield. With the AG 7000YG, RIB technology with 5.0 and $7.5 \%$ of nontransgenic seeds provided the best yields, significantly higher than the one from traditional refuge, which did not differ from the conventional control. In the RIB treatments, differences in damage intensities by Spodoptera frugiperda did not reflect in yield differences. Refuge in the bag is a viable alternative to the currently used refuge method.
\end{abstract}

Index terms: Spodoptera frugiperda, Zea mays, seed mixture, refuge in the bag, insect resistance, yieldgard.

\section{Introdução}

As pragas principais do milho (Zea mays L.) podem acarretar perdas de produtividade de até $60 \%$, dependendo da cultivar, do tipo de milho e da época em que ocorre o ataque (Cruz \& Turpin, 1983). Neste contexto, a utilização de cultivares transgênicas tem sido de extrema importância para o avanço na produtividade da cultura do milho. Na safra de 2010/2011, as cultivares transgênicas representaram $55,6 \%$ da área de milho cultivada no Brasil. No Brasil, o cultivo de culturas geneticamente modificadas (GM) atingiu 25,4 milhões de hectares na safra 2011/2012, o que tornou o país o segundo maior produtor de culturas GM, atrás apenas dos Estados Unidos, com 66,8 milhões de hectares (James, 2010).

Alguns insetos-alvo da tecnologia $\mathrm{Bt}$ podem desenvolver resistência e transmitir essa resistência para gerações futuras. O manejo da resistência de insetos (MRI) refere-se a um conjunto de práticas aplicadas nas áreas agrícolas, com vistas a evitar ou retardar a evolução da resistência das pragas aos

Pesq. agropec. bras., Brasília, v.47, n.7, p.886-891, jul. 2012 
métodos empregados no seu controle (Machado \& Fiuza, 2011).

Nesse contexto, a melhor maneira de preservar os benefícios das plantas transgênicas é a implementação de áreas de refúgio (Martinelli \& Omoto, 2005). Essa tecnologia preconiza a semeadura de área com híbrido milho não $\mathrm{Bt}$ - de porte e ciclo vegetativo similares aos do híbrido transgênico -, na área semeada com milho Bt. No refúgio, a praga-alvo terá condições de sobrevivência e reprodução, sem ser exposta à pressão de seleção da proteína inseticida expressa na planta Bt. O objetivo do refúgio é sincronizar os cruzamentos de possíveis adultos sobreviventes à proteína inseticida com insetos ainda suscetíveis provenientes da área de refúgio, num esforço de preservar na descendência dos cruzamentos a suscetibilidade à proteína transgênica, com consequente manutenção dos benefícios da tecnologia Bt (Bourguet et al., 2005).

Para facilitar o cumprimento das normas técnicas de implantação de refúgios, as principais empresas multinacionais produtoras de sementes de milho têm cogitado a possibilidade de misturar uma percentagem de sementes não transgênicas diretamente nos sacos com sementes transgênicas. Essa tecnologia foi denominada de refúgio no saco ("refuge in the bag" - RIB). Embora pareça ser uma solução simples para o problema, essa tecnologia não foi utilizada antes porque os híbridos existentes no mercado exigiam uma área muito grande de refúgio, de pelo menos $10 \%$. Como as sementes não transgênicas misturadas no saco de transgênicas podem resultar no desenvolvimento de plantas com desempenho agronômico inferior às demais, em razão da suscetibilidade ao ataque dos insetos e da ausência de controle químico, $10 \%$ pode representar um prejuízo elevado para os agricultores. No entanto, a nova geração de transgênicos resistentes a insetos apresentam duas proteínas Bt no mesmo híbrido, o que reduz a necessidade de refúgio para $5 \%$ da área. Ao se considerar que $95 \%$ da lavoura será pouco atacada, é provável que a mistura seja economicamente preferível à semeadura de $5 \%$ de não transgênicos à parte. Nos Estados Unidos, a Agência de Proteção Ambiental (EPA) aprovou a utilização da tecnologia de refúgio no saco para a safra de 2012, em híbridos com duas proteínas Bt.

Entre as vantagens dessa nova tecnologia, destacamse: manejo simplificado do refúgio, sem a necessidade de técnicas diferentes para cada parte da lavoura; uso de apenas um saco de sementes, o que evita eventuais problemas, como, por exemplo, mistura na proporção errada de sementes; conveniência, já que o agricultor terá menos trabalho na organização da semeadura; aumento da eficiência de semeadura, o que proporciona melhor aproveitamento do tempo e das condições no campo; certeza do cumprimento das normas de refúgio, pois as sementes de milho não $\mathrm{Bt}$ virão misturadas uniformemente no saco; menor perda de produtividade, uma vez que haverá somente 5\% de milho não $\mathrm{Bt}$; afrouxamento na pressão de seleção de indivíduos resistentes a inseticidas químicos, em razão da redução no seu uso; e melhor distribuição do refúgio, proporcionada pela melhor distribuição das plantas não Bt ao longo da área cultivada, o que aumenta a probabilidade de os indivíduos suscetíveis cruzarem com os que tenham adquirido resistência.

O objetivo deste trabalho foi comparar o uso de refúgio tradicional em áreas separadas com a tecnologia alternativa de refúgio pela mistura de sementes nãotransgênicas no saco ("refuge in the bag" - RIB) em diferentes proporções.

\section{Material e Métodos}

O experimento foi realizado na fazenda da Faculdade de Ciências Agrárias e Veterinárias, da Universidade Estadual Paulista, Jaboticabal, SP $\left(21^{\circ} 15^{\prime} 22^{\prime \prime S ~ e ~}\right.$ $48^{\circ} 18^{\prime} 58^{\prime \prime} \mathrm{W}$, a $595 \mathrm{~m}$ de altitude). O clima da região é do tipo Aw, conforme a classificação de Köppen, e a precipitação anual foi de $1.405 \mathrm{~mm}$, no ano agrícola de 2010/2011. Foram utilizadas sementes de quatro híbridos simples precoces de milho: AG 7000YG (transgênico), AG 7000 (convencional), DKB 390YG (transgênico) e DKB 390 (convencional), fornecidos pela empresa Monsanto do Brasil Ltda.

O experimento foi constituído por sete tratamentos para cada híbrido (Tabela 1): quatro tratamentos de RIB, com diferentes percentagens de mistura de sementes não transgênicas no saco $(2,5,5,0,7,5$ e $10 \%$ ); refúgio tradicional, com plantio de $10 \%$ da área exclusivamente com sementes não transgênicas; e uma área totalmente transgênica e outra totalmente convencional. Utilizou-se o delineamento em blocos ao acaso, com 19 repetições. As parcelas experimentais foram constituídas de quatro linhas de $20 \mathrm{~m}$ de comprimento cada, espaçadas em $0,9 \mathrm{~m}$ entre linhas e $0,2 \mathrm{~m}$ entre plantas, o que possibilitou população total aproximada de 55.555 plantas por hectare. 
As sementes transgênicas foram semeadas mecanicamente em sistema de plantio direto, enquanto as não transgênicas foram semeadas manualmente com uso de "matracas", de acordo com a mistura de sementes estipulada para cada tratamento. Não foi feita a aplicação de inseticidas durante o experimento, e as plantas não transgênicas não foram identificadas na área. A adubação e os tratos culturais foram feitos conforme as recomendações técnicas para a cultura do milho.

Para garantir a infestação com a lagarta-do-cartucho [Spodoptera frugiperda (J.E. Smith, 1797)] na área, foram feitas infestações artificiais de lagartas criadas no Laboratório de Resistência de Plantas a Insetos do Departamento de Fitossanidade, da Faculdade de Ciências Agrárias e Veterinárias, da Universidade Estadual Paulista, Jaboticabal, SP. As lagartas foram obtidas por meio da criação estoque, mantidas em laboratório e alimentadas com dieta artificial (Kasten Junior et al., 1978). As mariposas foram soltas ao acaso na área aos 3, 5, 10 e 15 dias após a semeadura (DAS).

Tabela 1. Proporção de sementes transgênicas (YG) e não transgênicas de híbridos de milho utilizadas nos tratamentos.

\begin{tabular}{lccccc}
\hline Tratamento & \multicolumn{3}{c}{ Percentagem } & & de sementes (\%) \\
\cline { 2 - 3 } \cline { 5 - 6 } & AG 7000YG & AG 7000 & & DKB 390YG & DKB 390 \\
\hline RIB, mistura 2,5\% & 97,5 & 2,5 & & 97,5 & 2,5 \\
RIB, mistura 5,0\% & 95,0 & 5,0 & & 95,0 & 5,0 \\
RIB, mistura 7,5\% & 92,5 & 7,5 & & 92,5 & 7,5 \\
RIB, mistura 10\% & 90,0 & 10,0 & & 90,0 & 10,0 \\
Refúgio tradicional & 90,0 & 10,0 & & 90,0 & 10,0 \\
Transgênico & 100 & 0 & & 100 & 0 \\
Convencional & 0 & 100 & & 0 & 100 \\
\hline
\end{tabular}

RIB, refúgio proporcionado pelo plantio de sementes convencionais e transgênicas, misturadas no saco de sementes ("refuge in the bag").
Foram feitas avaliações visuais de danos nas plantas e realizadas estimativas de produtividade. As avaliações visuais para S. frugiperda foram realizadas segundo escala de notas de dano nas plantas (Tabela 2), adaptada de Davis et al. (1992). As plantas foram avaliadas aos 17, 24, 31 e 38 DAS. A intensidade de dano nos tratamentos foi determinada pela avaliação do dano no cartucho das plantas. Avaliaram-se dez plantas na região central de cada linha da parcela, mas não necessariamente as mesmas plantas nas avaliações sucessivas. A nota de dano de cada parcela foi obtida pela média das 40 notas individuais. Para as estimativas de produtividade de grãos $\left(\mathrm{Mg} \mathrm{ha}^{-1}\right)$, colheram-se os 5,0 m centrais de cada uma das quatro linhas das parcelas, e corrigiu-se a umidade dos grãos para $13 \%$.

A análise de variância foi realizada com uso do programa SAS (SAS Institute, 2008), e as médias foram comparadas pelo teste $t$, a $5 \%$ de probabilidade.

\section{Resultados e Discussão}

Verificou-se a presença de $S$. frugiperda em plantas transgênicas e não transgênicas, em todos os tratamentos e híbridos. Contudo, lagartas grandes não foram visualizadas nas plantas transgênicas, o que pode ter sido efeito da mortalidade ou do atraso no desenvolvimento em razão da ingestão das toxinas $\mathrm{Bt}$ (Bokonon-Ganta et al., 2003).

A precisão experimental, estimada pelos coeficientes de variação, foi considerada elevada, com exceção das avaliações efetuadas aos 17 DAS. De modo geral, os tratamentos com ambos os híbridos mantiveram a mesma ordem em relação às notas de dano, ao longo

Tabela 2. Escala de notas ( 0 a 9) utilizadas para avaliação de danos por ataque de Spodoptera frugiperda no cartucho do milho $^{(1)}$.

\begin{tabular}{|c|c|}
\hline Nota & Descrição \\
\hline 0 & Planta sem injúria. \\
\hline 1 & Planta com pontuações. \\
\hline 2 & Planta com pontuações, 1 a 3 lesões circulares pequenas (até 1,5cm). \\
\hline 3 & Planta com 1 a 5 lesões circulares pequenas (até $1,5 \mathrm{~cm}$ ); mais 1 a 3 lesões alongadas (até 1,5 cm). \\
\hline 4 & Planta com 1 a 5 lesões circulares pequenas (até 1,5 cm); mais 1 a 3 lesões alongadas (entre 1,5 e $3,0 \mathrm{~cm}$ ); mais pequenos furos circulares (até $0,5 \mathrm{~cm}$ ). \\
\hline 5 & Planta com 1 a 3 lesões alongadas grandes $(>3,0 \mathrm{~cm})$ em 1 a 2 folhas; mais 1 a 5 furos ou lesões alongadas (até $1,5 \mathrm{~cm})$. \\
\hline 6 & Planta com 1 a 3 lesões alongadas grandes $(>3,0 \mathrm{~cm})$ em 2 ou mais folhas; mais 1 a 3 furos grandes $(>1,5 \mathrm{~cm})$ em 2 ou mais folhas. \\
\hline 7 & Planta com 3 a 5 lesões alongadas grandes $(>3,5 \mathrm{~cm})$ em 2 ou mais folhas; mais 3 a 5 furos grandes $(>1,5 \mathrm{~cm})$ em 2 ou mais folhas. \\
\hline 8 & $\begin{array}{l}\text { Planta com muitas lesões alongadas (mais de 5) de todos os tamanhos na maioria das folhas. Muitos furos médios a grandes (mais de 5) maiores que } \\
\qquad 3,0 \mathrm{~cm} \text { em muitas folhas. }\end{array}$ \\
\hline 9 & Planta com folhas quase totalmente destruídas. \\
\hline
\end{tabular}

${ }^{(1)}$ Adaptada de Davis et al. (1992). 
das avaliações (Tabela 3). O tratamento com área totalmente convencional, não transgênica, apresentou maior intensidade de dano, com notas de 2,95, 6,03, 7,94 e 7,99 para o híbrido AG 7000 e notas de 2, 6, 7 e 8 para o híbrido DKB 390, respectivamente para as avaliações aos 17, 24, 31 e 38 DAS. Observou-se que, aos 38 DAS, as notas dos dois híbridos para esse tratamento não diferiram significativamente e que, a nota de dano aumentou em 2,7 vezes, entre a primeira e a última avaliação no híbrido AG 7000, e em quatro vezes no DKB 390. Proporcionalmente, os demais tratamentos tiveram aumentos mais expressivos da primeira para a última avaliação; porém, as notas de dano foram inferiores em razão da menor intensidade de ataque da lagarta-do-cartucho.

Ao se comparar os dois híbridos, verificaram-se intensidades de dano significativamente diferentes (Tabela 3), exceto para o tratamento inteiramente convencional e para o com mistura de $10 \%$. O tratamento totalmente transgênico, como esperado, apresentou a menor intensidade de dano, com notas de 3,88 e 3,38 para os híbridos AG 7000YG e DKB 390YG, respectivamente. De acordo com Soberón et al. (2009), a toxina Bt é expressa continuamente nos tecidos da planta, o que explica o eficácia de controle dessa tecnologia. Os baixos valores de notas obtidas neste trabalho com o tratamento totalmente transgênico concordam com relatos da literatura (Williams et al., 1997; Buntin et al., 2001; Waquil et al., 2002; Fernandes et al., 2003; Michelotto et al., 2011).

Observou-se que, aos 38 DAS, os tratamentos convencional e com $10 \%$ de mistura apresentaram as maiores notas (Tabela 3), seguidos do tratamento com refúgio tradicional, que não diferiu significativamente do tratamento com $7,5 \%$ de mistura no híbrido AG 7000YG mas apresentou maior nota com o híbrido DKB 390YG. Nos demais tratamentos, observaramse reduções significativas nas notas de dano, a cada redução na proporção de sementes não transgênica.

$\mathrm{Na}$ área de refúgio tradicional, onde somente há milho não transgênico, a probabilidade de sobrevivência e multiplicação da lagarta-do-cartucho é muito alta, e a praga pode completar vários ciclos durante o ciclo da cultura. No entanto, quando se utiliza mistura de sementes, somente sobreviverão as lagartas que acertarem, ao acaso, as plantas não transgênicas, pois não há evidência de que elas consigam distinguir as folhas de milho Bt e não Bt com base nos compostos voláteis das plantas. Porém, sabe-se que as lagartas apresentam não preferência para alimentação em milho Bt quando expostas ao contato físico com o hospedeiro (Mendes et al., 2011). As toxinas Bt são deterrentes para as larvas de lepidópteros, e essas larvas podem evitar plantas com toxinas Bt quando existem áreas de refúgio próximas. Contudo, os primeiros instares das larvas são mais sensíveis aos efeitos da toxina, o que reduz a migração de larvas do milho $\mathrm{Bt}$ para o não $\mathrm{Bt}$ (Dutton et al., 2005). Assim, a mistura de sementes proporciona, ao mesmo tempo, controle satisfatório da praga e sobrevivência de uma quantidade de indivíduos suscetíveis que podem reproduzir-se, o que diminui a pressão de seleção a favor de indivíduos resistentes e contribui com as práticas de MIP e MRI.

A precisão experimental também foi considerada elevada para os dados de produtividade. Para todos os tratamentos avaliados, a produtividade foi maior com o híbrido DKB 390YG (Tabela 4). As médias de produtividade dos tratamentos com esse híbrido não

Tabela 3. Notas de dano obtidas aos 17, 24, 31 e 38 dias após a semeadura (DAS), nos híbridos de milho AG 7000YG e DKB $390 \mathrm{YG}$, nos diferentes tratamentos ${ }^{(1)}$.

\begin{tabular}{|c|c|c|c|c|c|c|c|c|}
\hline \multirow[t]{2}{*}{ Tratamento } & \multicolumn{2}{|c|}{17 DAS } & \multicolumn{2}{|c|}{24 DAS } & \multicolumn{2}{|c|}{31 DAS } & \multicolumn{2}{|c|}{$38 \mathrm{DAS}$} \\
\hline & AG 7000YG & DKB 390YG & AG 7000YG & DK B390YG & AG 7000YG & DKB 390YG & AG 7000YG & DKB 390YG \\
\hline Convencional & $2,95 \mathrm{aA}$ & $2,00 \mathrm{bA}$ & $6,03 \mathrm{aA}$ & $6,00 \mathrm{aA}$ & $7,94 \mathrm{aA}$ & $7,00 \mathrm{bA}$ & 7,99aA & $8,00 \mathrm{aA}$ \\
\hline RIB, mistura $10 \%$ & $1,32 \mathrm{aBC}$ & $0,74 \mathrm{bC}$ & $3,37 \mathrm{bB}$ & $3,63 \mathrm{aB}$ & $4,39 \mathrm{aB}$ & $4,71 \mathrm{bB}$ & $5,41 \mathrm{aB}$ & $5,24 \mathrm{aB}$ \\
\hline Refúgio tradicional & $1,51 \mathrm{aB}$ & $0,99 \mathrm{bB}$ & $3,23 \mathrm{aBC}$ & $3,43 \mathrm{aBC}$ & $4,45 \mathrm{aB}$ & $4,53 \mathrm{aCB}$ & $5,10 \mathrm{aC}$ & $4,75 \mathrm{bC}$ \\
\hline RIB, mistura 7,5\% & $1,22 \mathrm{aCD}$ & $0,78 \mathrm{bC}$ & $2,96 \mathrm{bCD}$ & $3,34 \mathrm{aC}$ & $4,19 \mathrm{aBC}$ & $4,37 \mathrm{aC}$ & $4,90 \mathrm{aC}$ & $4,51 \mathrm{bD}$ \\
\hline RIB, mistura $5,0 \%$ & $1,24 \mathrm{aCD}$ & $0,67 \mathrm{bCD}$ & $2,92 \mathrm{bD}$ & $3,25 \mathrm{aC}$ & $3,99 \mathrm{aCD}$ & $4,01 \mathrm{aD}$ & $4,55 \mathrm{aD}$ & $4,16 \mathrm{bE}$ \\
\hline RIB, mistura $2,5 \%$ & $1,12 \mathrm{aCD}$ & $0,63 \mathrm{bCD}$ & $2,62 \mathrm{bE}$ & $2,94 \mathrm{aD}$ & $3,73 \mathrm{aD}$ & $3,57 \mathrm{aE}$ & $4,16 \mathrm{aE}$ & $3,69 \mathrm{bF}$ \\
\hline RIB, mistura Bt & $1,02 \mathrm{aD}$ & $0,53 \mathrm{bD}$ & $2,42 \mathrm{bE}$ & $2,81 \mathrm{aD}$ & $3,38 \mathrm{aE}$ & $3,41 \mathrm{aE}$ & $3,88 \mathrm{aF}$ & $3,38 \mathrm{bG}$ \\
\hline $\mathrm{CV}(\%)$ & 25,00 & 32,73 & 12,93 & 9,71 & 9,72 & 7,86 & 7,97 & 6,38 \\
\hline
\end{tabular}

${ }^{(1)}$ Médias seguidas de letras iguais, minúsculas nas linhas e maiúsculas nas colunas, não diferem entre si pelo teste t, a $5 \%$ de probabilidade, para cada época de avaliação. RIB, refúgio proporcionado pelo plantio de sementes convencionais e transgênicas, misturadas no saco de sementes ("refuge in the bag"). 
Tabela 4. Produtividade média $\left(\mathrm{Mg} \mathrm{ha}^{-1}\right)$ dos híbridos de milho DKB 390YG e AG 7000YG, nos diferentes tratamentos ${ }^{(1)}$.

\begin{tabular}{lcc}
\hline Tratamento & DKB 390YG & AG 7000YG \\
\hline RIB, mistura 7,5\% & $10,43 \mathrm{aA}$ & $9,50 \mathrm{bA}$ \\
RIB, mistura 5,0\% & $10,46 \mathrm{aA}$ & $9,47 \mathrm{bA}$ \\
Transgênico & $10,63 \mathrm{aA}$ & $9,24 \mathrm{bAB}$ \\
RIB, mistura 2,5\% & $10,53 \mathrm{aA}$ & $9,41 \mathrm{bAB}$ \\
RIB, mistura 10\% & $10,49 \mathrm{aA}$ & $9,35 \mathrm{bAB}$ \\
Refúgio tradicional & $10,30 \mathrm{aA}$ & $8,92 \mathrm{bBC}$ \\
Convencional & $9,20 \mathrm{aB}$ & $8,58 \mathrm{bC}$ \\
\hline CV $(\%)$ & 6,49 & 8,86
\end{tabular}

${ }^{(1)}$ Médias seguidas de letras iguais, minúsculas nas linhas e maiúsculas nas colunas, não diferem entre si pelo teste t, a $5 \%$ de probabilidade. RIB, refúgio proporcionado pelo plantio de sementes convencionais e transgênicas, misturadas no saco de sementes ("refuge in the bag").

diferiram significativamente entre si, com exceção do tratamento inteiramente convencional que apresentou menor produtividade.

Para o híbrido AG 7000YG, os tratamentos com mistura foram os mais produtivos e não diferiram entre si (Tabela 4). O tratamento com refúgio tradicional, neste híbrido, apresentou menor produtividade que os tratamentos com 5,0 e 7,0\% de mistura, e não diferiu significativamente das demais misturas nem dos tratamentos inteiramente transgênico ou inteiramente convencional.

Para que um híbrido transgênico expresse totalmente o seu potencial, recomenda-se, como medida adicional de controle, a aplicação de inseticidas quando $20 \%$ das plantas atingirem a nota de dano 4 (Lourenção et al., 2009). Nos tratamentos com mistura, apesar de teremse observado intensidades de dano significativamente diferentes em quase todos os tratamentos, as médias de produtividade não diferiram em nenhum dos híbridos. Esse resultado indica que há necessidade de mais estudos sobre o nível de controle ideal com o emprego da tecnologia de refúgio no saco.

Os resultados obtidos no presente trabalho são indicativos de que mais estudos, com foco principalmente na biologia, no ciclo de vida e no comportamento de $S$. frugiperda, são necessários para o melhor aproveitamento e manejo da tecnologia RIB.

\section{Conclusões}

1. A tecnologia de refúgio no saco (RIB) é alternativa interessante e deve ser considerada para substituir o método de refúgio tradicional utilizado atualmente, na tentativa de retardar a evolução da resistência ao Bt nas principais pragas da cultura do milho.

2. A intensidade de danos, de maneira geral, diminui com a diminuição na proporção de sementes não transgênicas usadas na tecnologia RIB, mas isso não se reflete em diferenças na produtividade.

3. Os híbridos influenciam na eficácia da tecnologia RIB.

\section{Agradecimentos}

À Empresa Monsanto do Brasil Ltda., pelo fornecimento das sementes de milho.

\section{Referências}

BOKONON-GANTA, A.H.; BERNAL, J.S.; PIETRANTONIO P.V.; SÉTAMOU, M. Survivorship and development of fall armyworm, Spodoptera frugiperda (J. E. Smith) (Lepidoptera: Noctuidae), on conventional and transgenic maize cultivars expressing Bacillus thuringiensis Cry9C and Cry1A(b) endotoxins. International Journal of Pest Management, v.49, p.169-175, 2003.

BOURGUET, D.; DESQUILBET, M.; LEMARIE, S. Regulating insect resistance management: the case of non-Bt corn refuges in the US. Journal of Environmental Management, v.76, p.210-220, 2005.

BUNTIN, G.D.; LEE, R.D.; WILSON, D.M.; MCPHERSON, R.M. Evaluation of YieldGard transgenic resistance for control of fall armyworm and corn earworm (Lepidoptera: Noctuidade) on corn. Florida Entomologist, v.84, p.37-42, 2001.

CRUZ, I.; TURPIN, F.T. Yield impact of larval infestations of the fall armyworm (Lepidoptera, Noctuidae) to midwhorl growth stage of corn. Journal of Economic Entomology, v.76, p.1052-1054, 1983.

DAVIS, F.M.; NG, S.S.; WILLIAMS, W.P. Visual rating scales for screening whorl-stage corn for resistance to fall armyworm. Mississippi: Mississippi State University, 1992. 9p. (MAFES. Technical bulletin, 186).

DUTTON,A.; ROMEIS, J.; BIGLER, F. Effects of Bt maize expressing $\mathrm{Cry} 1 \mathrm{Ab}$ and $\mathrm{Bt}$ spray on Spodoptera littoralis. Entomologia Experimentalis et Applicata, v.114, p.161-169, 2005.

FERNANDES, O.D.; PARRA, J.R.P.; F. NETO, A.; PÍCOLI, R.; BORGATTO, A.F.; DEMÉTRIO, C.G.B. Efeito do milho geneticamente modificado mon810 sobre a lagarta-do-cartucho Spodoptera frugiperda (J. E. Smith, 1797) (Lepidoptera: Noctuidae). Revista Brasileira de Milho e Sorgo, v.2, p.25-35, 2003.

JAMES, C. Global status of commercialized Biotech/GM Crops: 2010. New York: ISAAA, 2010. 30p. (ISAAA Brief, 42).

KASTEN JUNIOR, A.A.; PRECETTI, C.M.; PARRA, J.R.P. Dados biológicos comparativos de Spodoptera frugiperda (J.E. Smith, 1797) em duas dietas artificiais e substrato natural. Revista de Agricultura, v.53, p.68-78, 1978. 
LOURENÇÃO, A.L.F.; BARROS, R.; MELO, E.P. de. Milho Bt: uso correto da tecnologia. In: TECNOLOGIA e produção: milho safrinha e culturas de inverno. 5.ed. Maracaju: Fundação MS, 2009. p.79-89.

MACHADO, V.; FIUZA, L.M. Manejo da resistência: na era das plantas transgênicas. Oecologia Australis, v.15, p.291-302, 2011.

MARTINELLI, S.; OMOTO, C. Resistência de insetos a plantas geneticamente modificadas. Biotecnologia Ciência e Desenvolvimento, v.34, p.67-77, 2005.

MENDES, S.M.; BOREGAS, K.G.B.; LOPES, M.E.; WAQUIL, M.S.; WAQUIL, J.M. Respostas da lagarta-do-cartucho a milho geneticamente modificado expressando a toxina Cry $1 \mathrm{~A}(\mathrm{~b})$. Pesquisa Agropecuária Brasileira, v.46, p.239-244, 2011.

MICHELOTTO, M.D.; FINOTO, E.L.; MARTINS, A.L.M.; DUARTE, A.P. Interação entre transgênicos $(B t)$ e inseticidas no controle de pragas-chave em híbridos de milho-safrinha. Arquivos do Instituto Biológico, v.78, p.71-79, 2011.

SAS INSTITUTE. SAS/STAT user's guide. Version 9.1.3. Cary: SAS Institute, 2008.

SOBERÓN, M.; GILL, S.S.; BRAVO, A. Signaling versus punching hole: how do Bacillus thuringiensis toxins kill insect midgut cells? Cellular and Molecular Life Sciences, v.66, p.1337-1349, 2009.

WAQUIL, J.M.; VILLELA, F.M.F.; FOSTER, J.E. Resistência do milho (Zea mays L.) transgênico (Bt) à lagarta-do-cartucho, Spodoptera frugiperda (Smith) (Lepidoptera: Noctuidae). Revista Brasileira de Milho e Sorgo, v.1, p.1-11, 2002.

WILLIAMS, W.P.; SAGERS, J.B.; HANTEN, J.A.; DAVIS, F.M.; BUCKLEY, P.M. Transgenic corn evaluated for resistance to fall armyworm and southwestern corn borer. Crop Science, v.37, p.957-962, 1997.

Recebido em 26 de janeiro de 2012 e aprovado em 12 de março de 2012 